\title{
Instrumentation des phases initiales du projet architectural pour le choix des matériaux
}

Informing the First Stages in the Architectural Process on Matters Concerning Choices of Materials

\section{Nathalie Tornay}

\section{(2) OpenEdition}

\section{Journals}

Édition électronique

URL : http://journals.openedition.org/crau/581

DOI : $10.4000 /$ crau. 581

ISSN : 2547-5746

\section{Éditeur}

Éditions du patrimoine

Édition imprimée

Date de publication : 1 novembre 2012

Pagination : 202-209

ISBN : 978-2-7577-0108-9

ISSN : 1296-4077

\section{Référence électronique}

Nathalie Tornay, «Instrumentation des phases initiales du projet architectural pour le choix des matériaux », Les Cahiers de la recherche architecturale et urbaine [En ligne], 26/27 | 2012, mis en ligne le 01 novembre 2017, consulté le 03 mai 2019. URL : http://journals.openedition.org/crau/581 ; DOI : 10.4000/crau.581 
Ces recherches questionnent l'intégration des dimensions techniques, écologiques et sensibles des matériaux dans des outils d'aide à la conception pour la contribution à la genèse d'idées fondatrices, à la définition d'un ou des parti(s), ou concept(s) propres aux phases initiales de projet.

\section{Instrumentation des phases initiales du projet architectural pour le choix
des matériaux}

NATHALIE TORNAY

Le choix des matériaux à l'échelle architecturale, urbaine ou paysagère définit le caractère du projet. Qu'il s'agisse de la surface des matériaux, qui traduit un parti d'insertion dans l'existant, de l'affirmation des volontés architecturales ou encore de la mise en valeur de contrastes, ou qu'il s'agisse des matériaux invisibles (isolation) qui traitent des notions de confort thermique ou acoustique : le choix des matériaux intègre une dimension sensible. Cependant, ce choix résulte d'une réflexion sur de nombreux critères. Les matériaux possèdent différentes dimensions. La dimension technique traite la mise en œuvre des matériaux par les aspects constructifs, structurels, etc. qui comprend les aspects normatifs. La dimension écologique développe une démarche environnementale dans le choix des matériaux en prenant en compte leur cycle de vie, leurs performances thermiques ou acoustiques, les matériaux locaux, etc. Elle soulève les aspects économiques. Par exemple le choix de matériaux locaux a pour conséquence la limitation des transports, ou encore les performances énergétiques

1. Nous entendons par équipe de concepteurs les acteurs de la conception architecturale, urbaine et du paysage, soit l'architecte ou l'urbaniste ou le paysagiste, le programmiste, l'ingénieur du bureau d'études ou du bureau de contrôle, le maître d'ouvrage et l'usager.

2. Développé par le Centre scientifique et technique du bâtiment (CSTB),

Élodie est un logiciel en ligne qui permet d'évaluer la performance environnementale d'un bâtiment sur tout son cycle de vie : http://ese2.cstb.fr/elodie/.

3. Pleiades+Comfie est un logiciel de simulation thermique dynamique bâtiment :

http://www.izuba.fr/logiciel/ pleiadescomfie. 
qui diminuent les consommations (de chauffe par exemple). Enfin leur coût est un critère, une contrainte permanente, pour les équipes de concepteurs ${ }^{1}$ dans le choix des matériaux. Dans ce contexte, se développent aujourd'hui de nombreux outils d'aide à la conception, des logiciels qui s'axent principalement sur la dimension environnementale et/ou technique des matériaux comme Elodie ${ }^{2}$, Pléiades+Comfie ${ }^{3}$, Equer ${ }^{4}$, Green Building Studio ${ }^{5}$, Cocon ${ }^{6}$, etc.

Nos travaux de recherche ${ }^{7}$ s'orientent vers une réflexion sur un outil d'aide à la conception dans le choix des matériaux qui opte pour l'intégration de la dimension sensible. Pour ce faire, il s'agit de relever, d'analyser les mécanismes cognitifs, intellectuels, sensoriels qui permettent aux concepteurs de recevoir et d'assimiler des informations sur les matériaux (dimension écologique et technique) à partir d'une approche sensible.

\section{Enjeux dès les phases initiales de projet}

Pour répondre à l'ambition de notre projet, nous devons étudier les modes opératoires des futurs utilisateurs, ce qui pose la question de l'intégration de notre outil dans la pratique des concepteurs. Notre outil se destine aux premières phases de réflexion sur le projet. C'est une phase qui se caractérise par son approche "de la précision imprécise ${ }^{8} »$. Nous nous appuyons sur les approches cognitives dans le champ de la conception pour identifier des procédures?. Le processus de conception est un contexte de résolution de problèmes qui est composé de trois phases successives ${ }^{10}$ :

- une phase de formulation d'un énoncé dont les composantes sont les finalités (type de projet), les utilités (type de programme), les performances (techniques, économiques) et l'intention des concepteurs et des acteurs.

- une phase de formulation de solutions. Elle correspond à la succession de propositions de formes, de partis, etc.

- une phase de concrétisation de solutions où les contraintes physiques ont un rôle déterminant (parti constructif)

En amont des phases de conception, les équipes de concepteurs disposent de peu d'informations. Pourtant pendant ces phases initiales, les choix fonctionnels, les choix d'implantation, d'orientation, de morphologie, etc. se décident. C'est le paradoxe de la conception architecturale ${ }^{11}$. Ce paradoxe intervient aussi à l'échelle du choix des matériaux où les décisions prises dans les phases initiales de projets sont indissociables du choix des matériaux.

\section{Les précédents dans un processus créatif}

Pour approfondir les connaissances sur les matériaux (leur dimension technique, écologique ou sensible), notre premier axe de recherche s'oriente vers la considération de notre héritage architectural, urbain et paysager. II s'agit de relever, d'étudier des précédents. Les précédents sont des projets existants à l'échelle architecturale, urbaine ou paysagère, qui intègrent des qualités remarquables dans le choix des matériaux.

Le principal atout des précédents dans le choix des matériaux est d'avoir le potentiel de présenter, représenter, d'analyser l'ensemble des dimensions des matériaux. Ils sont des vecteurs de connaissances.
4. Equer est un outil d'évaluation de la qualité environnementale des bâtiments, ayant pour but d'aider les acteurs à mieux cerner les conséquences de leurs choix: http://www.izuba.fr/logiciel/equer.

5. Le service Web Autodesk® Green Building Studio $®$ propose des analyses de la consommation d'énergie, d'eau et des émissions de $\mathrm{CO}_{2}$ des bâtiments sur la base des données météorologiques locales afin d'évaluer la consommation potentielle de ressources: http://usa.autodesk.com/adsk/ servlet/pc/ index?id=11179508\&sitelD=123112.

6. C'est un logiciel d'estimation de la qualité environnementale de bâtiment, afin de comparer des solutions constructives en termes technique et environnemental : http://www.eosphere.fr/cocon.

7. Cet article est issu de la thèse de doctorat en génie civil, soutenue le 21 mars 2011 (dir. Luc Adolphe et Frédéric Bonneaud) à l'Insa de Toulouse, école doctorale "Mécanique, énergétique, génie civil et procédés (Toulouse), en partenariat avec le laboratoire de Matériaux et durabilité des constructions, LMDC; laboratoire d'accueil LRA, laboratoire de recherche en architecture de l'Énsa de Toulouse. Titre de la thèse: Vers des outils d'aide à la conception pour intégrer les dimensions techniques, écologiques et sensibles des matériaux de construction. 
À toutes les échelles, les précédents ont le potentiel d'être exposés selon une description générale, une analyse à partir de concepts à l'échelle architecturale ${ }^{12}$ ou à l'échelle du paysage ${ }^{13}$, etc. Chaque précédent peut être décliné à l'échelle du projet selon les trois principales approches :

- l'approche technique est étudiée selon les choix constructifs et les types d'enveloppes ;

- l'approche écologique évalue le projet selon quatre critères actuellement retenus (performance thermique, performance acoustique, implantation, compacité) ;

- l'approche sensible considère les volontés architecturales, en classant les projets selon les choix constructifs et les pourcentages de surface vitrée et en définissant le rôle des matériaux (marquage, contournement, franchissement, etc.).

À l'échelle des matériaux, les caractéristiques des matériaux sont développées :

- la dimension écologique évalue le projet selon six critères actuellement retenus (cycle de vie, performance thermique, performance acoustique, transport, santé, entretien) ;

- les dimensions techniques et sensibles se mêlent car les données physiques de matériaux permettent de caractériser les modalités sensorielles. Par exemple le facteur de réflexion $\rho$, permet d'estimer l'effet de la brillance d'un matériau, ou encore le coefficient d'absorption permet d'apprécier la résonnance/absorption des sons par un matériau, etc. II est question des critères suivants : brillance/ mât, lourd/léger, chaud/froid, rugueux/lisse, clair/foncé, etc.

L'étude des précédents a la capacité de considérer l'ensemble des caractéristiques des matériaux. Chaque précédent est codé ${ }^{14}$ selon les critères analysés. Par conséquent, ils forment un important corpus, ce système permet d'identifier (description), de comparer (analyse), et d'évaluer (étude des performances) les précédents dans le choix des matériaux.

\section{Choix des matériaux dans un lieu et un temps}

Les précédents permettent de transmettre des savoirs et des savoir-faire, de partager des réflexions sur le choix des matériaux. Cependant, leurs effets, leurs expressions, leurs vieillissements résultent d'un contexte environnant. Les facteurs climatiques (pluie, vent, température, ensoleillement), leur localisation (pollution atmosphérique), leur implantation (masques, orientation, etc.) jouent un rôle déterminant dans l'expression et par conséquent la perception des matériaux. Notre deuxième axe de recherche mesure donc l'importance du lieu dans le choix des matériaux.

L'idée de genius loci est empruntée aux Grecs, pour qui le lieu évoque les qualités inaltérables ou l'essence d'un endroit, associé à une divinité ou un esprit local. Au $\mathrm{XVIII}^{\mathrm{e}}$ siècle, le poète anglais Alexander Pope conseille aux architectes britanniques de " consulter le génie du lieu pour tout », ce qui aujourd'hui est devenu «l'analyse du site ${ }^{15}$ ». L'analyse du site a le potentiel de guider les équipes de concepteurs dans le choix des matériaux :

- les matériaux extraits ou fabriqués localement. II s'agit de considérer la culture locale, sa spécificité, ses caractéristiques :

- une approche chromatique (le bâti existant, la présence de végétation, la couleur de la terre, etc.) peut
8. Jean-Charles Lebahar, Le dessin d'architecte. Simulation graphique et réduction d'incertitude, Marseille, Éditions Parenthèse, 1983, p. 56

9. Nathalie Tornay, Frédéric Bonneaud, Luc Adolphe, "The Choice of Materials in Architecture: the influence of cognitive and cultural schemas of ecology », dans Claude Demers, André Potvin, Architecture Energy and the Occupant's Perspective. Proceedings of the 26th International
Conference on Passive and Low Energy Architecture, 22-24 June 2009, Quebec City, Canada, Presses Universitaires de Laval, p. 579-584.

10. Robert Prost, Conception architecturale, une investigation méthodologique, Paris, L'Harmattan, 1992, p. 43 et 86.

11. Luc Adolphe, L'intégration des connaissances techniques dans le processus de conception architecturale, Habilitation à diriger des recherches, Toulouse, université Paul Sabatier, 1995.

12. Pierre Fernandez, Guilerme Lassance, Des cibles aux intentions : penser les rapports entre qualité environnementale et qualité architecturale, rapport PUCA, 2003.

13. Liat Margolis, Alexander Robinson, Systèmes vivants et paysage : technologies et matériaux évolutifs, Bâle, Birkhäuser, 2008. 
influencer le choix des matériaux ${ }^{16}$. Avec une logique de " mimétisme », ou une logique qui joue sur I'harmonie des couleurs (contraste, couleur complémentaire). Les matériaux locaux ou issus de la production industrielle présentent des propriétés chromatiques propres, intrinsèques. II s'agit alors de relever les matériaux qui présentent les caractéristiques chromatiques recherchées ;

- les données climatiques, comme l'ensoleillement qui détermine une ambiance lumineuse peut créer des effets de matière, ou nuire à la pérennité des façades (salissures). Cela dépend de l'implantation et de la morphologie du projet. L'orientation des façades et le contexte environnant permettent des jeux sur la perception des façades, des traitements de sol ou encore sur les choix de revêtements intérieurs. La vision permet de réguler les effets de brillance, de translucidité, de clarté, etc. Le toucher permet d'apprécier la température de la surface, sa rugosité, etc. Enfin, la surface des matériaux peut jouer sur l'ouie en proposant des effets de résonnance, ou au contraire d'absorption. Sans oublier les caractéristiques olfactives de certains matériaux.

\section{Vers une proposition d'un outil d'aide à la conception}

Nos deux axes de recherche (les précédents et l'analyse du site) nous ont conduit à la construction d'un prototype d'outil d'aide à la conception dans le choix des matériaux, il se nomme dilem'matériaux. dilem'matériaux se décline en trois outils qui évoluent progressivement entre I'utilisation de précédents vers l'analyse de site.

La première entrée est un moteur de recherche par image. Comme le montre la figure 1, l'interface d'accueil est composée d'un mur d'images. II s'agit d'une base de données constituée d'images qui représentent des plans, coupes, façades, croquis, axonométries, photographies, etc. de précédents. Chaque image est indexée selon les caractéristiques écologiques, techniques et sensibles des matériaux. À chaque sélection, dilem'matériaux propose de nouvelles images en adéquation avec l'image sélectionnée. À tout moment, il est possible d'affiner les recherches en intégrant des objectifs environnementaux (performances thermiques, performances acoustiques, compacité, implantation, cycle de vie des matériaux, impact sur la santé, entretien et le transport des matériaux), ou des propriétés sensorielles des matériaux (lisse/rugueux, chaud/froid, brillant/mât, clair/foncé, etc.). La deuxième interface propose des images classées selon quatre niveaux ; la représentation d'un matériau (photographie de surfaces de matériaux), des caractéristiques techniques (type de structure, matériau de structure, etc.) des caractéristiques sensibles (concept, rôle des matériaux, etc.) et d'autres images issues du même précédent. Des mots clés sont eux aussi classés selon ces quatre niveaux. Ils correspondent à l'indexation des images présentes sur l'interface, et permettent d'affiner la recherche en sélectionnant directement un matériau ou un concept, etc. Enfin, l'interface des résultats conserve la même classification avec l'accès à des fiches matériaux qui présentent et caractérisent les matériaux issus de la recherche, des fiches actions qui analysent l'enjeu des matériaux des images sélectionnées. La dimension technique développe les questions de mise en œuvre, de structure, etc. La dimension sensible analyse le choix des matériaux par rapport au site, leurs rôles, leurs significations (marquages,

14. Umberto Eco, Sémiotique du message visuel, Paris, Le Seuil, 1970.

15. Richard Weston, Formes et matériaux dans I'architecture, Paris, Le Seuil, 2003, p. 100.

16. Marie-Pierre Servantie, Chromo-architecture, Paris, Éditions Alternatives, 2007. 
franchissement, mémoire, référence au tissage, etc.). Puis les fiches précédents présentent des projets architecturaux, urbains ou du paysage concernés par les qualités de matérialité retenues. II existe de fortes interactions entre ces fiches, elles font l'objet de nombreux liens de type hypertexte. L'objectif est de permettre une libre navigation entre l'ensemble des connaissances sur les matériaux. Ainsi, l'utilisateur sélectionne et enregistre uniquement les éléments qu'il estime en adéquation aves ses projets.

Actuellement, une maquette informatique de cette recherche par image est en cours d'élaboration. Elle permettra de tester l'efficience des choix des images des précédents, l'efficacité de leur indexation, la présentation des résultats, etc. et de les améliorer.

La deuxième entrée intègre une analyse de site et l'étude de précédents. Elle se nomme Chroma. Elle est une aide aux choix des matériaux à partir d'une étude chromatique de site, sa navigation est schématisée sur la figure 2. II s'agit de télécharger une ou plusieurs photographie(s) du site. Ce qui permet de choisir l'échelle d'étude (une photographie de la région, de la ville, de la parcelle, des constructions adjacentes, du sol,

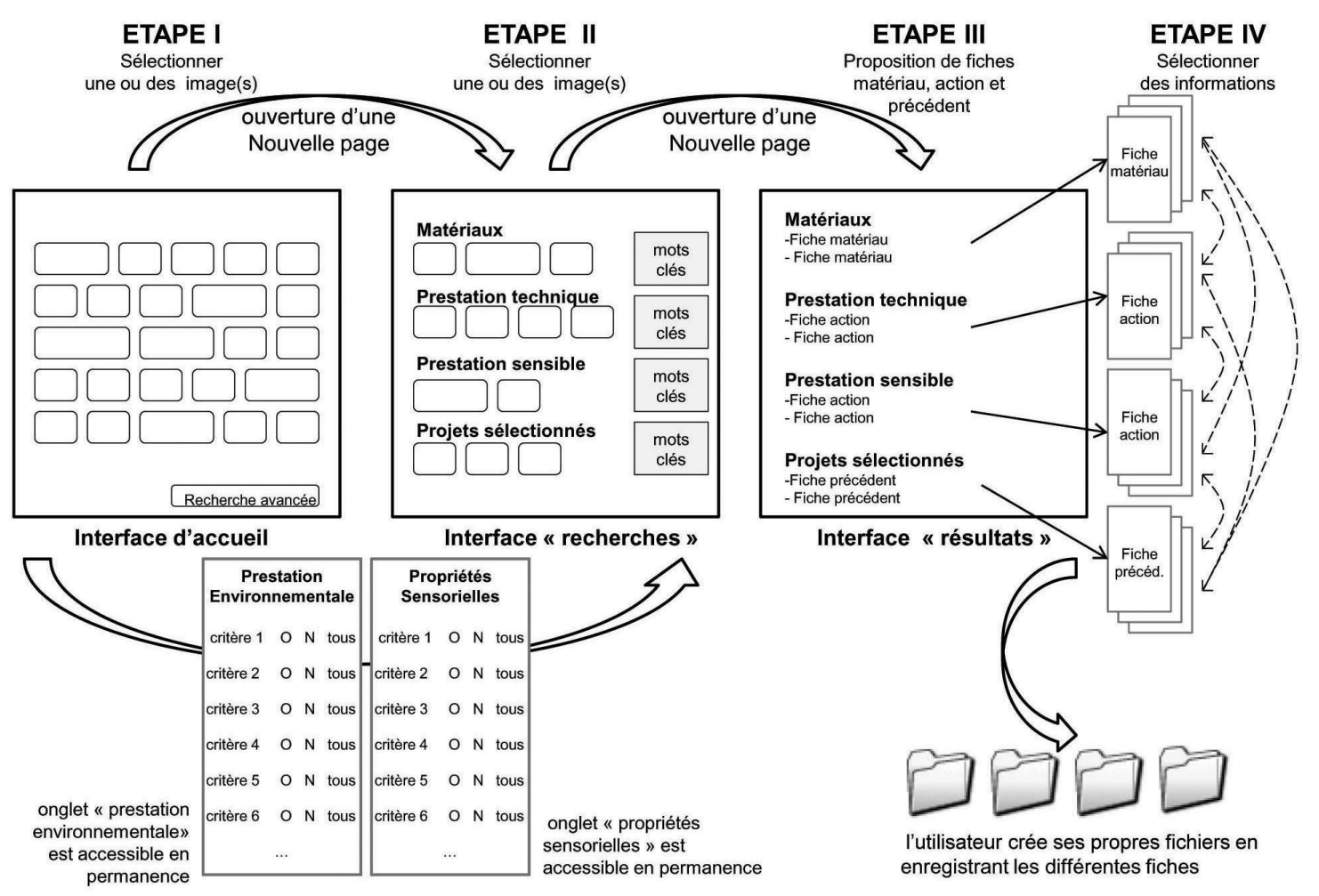

Fig. 1.

Schéma de la navigation de l'outil recherche par image. 
d'un détail, etc.). À partir de cette ou ces photographie(s) apparaît un diagnostic représenté par un nuancier. $\mathrm{Ce}$ nuancier permet de proposer trois palettes avec des couleurs qui proposent une attitude de mimétisme, d'intégration ou de dynamisme. Chacune de ces palettes oriente vers des matériaux possédant les mêmes propriétés chromatiques. Les fiches matériaux sont alors disponibles. Elles possèdent toujours les liens avec les fiches actions et les fiches précédents. La sélection de matériau peut toujours être affinée selon la dimension environnementale et les propriétés sensorielles. Ainsi, comme précédemment, I'utilisateur navigue librement et enregistre uniquement les éléments qu'il estime en adéquation avec ses projets.

Enfin, la troisième entrée est dédiée aux projets des utilisateurs, sa navigation est schématisée par la figure 3. Elle se nomme Chrono, son objectif est de tester différentes variantes des projets dans le temps. Tout d'abord il s'agit de télécharger la volumétrie, I'implantation du projet à partir de logiciels comme SketchUp ${ }^{17}$. S'ensuit un court questionnaire qui décrit le contexte environnant (climat, pollution atmosphé-
ETAPE

Télécharger une photographie
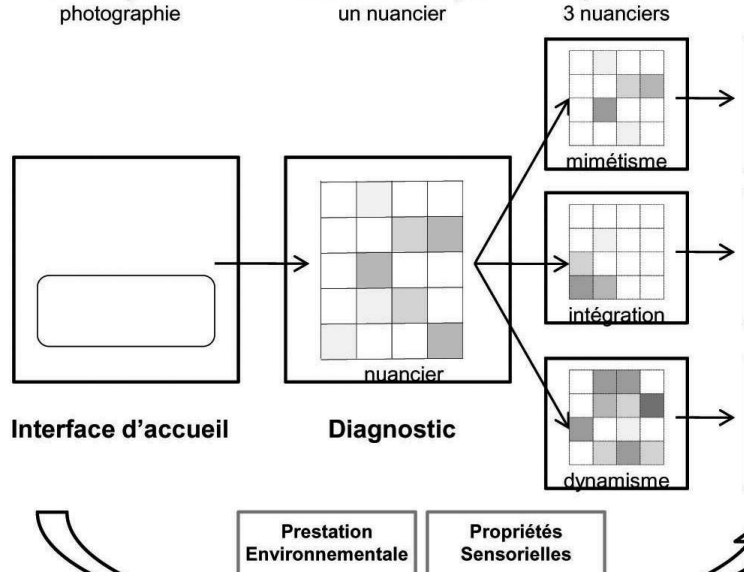

1

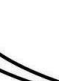
environnementale» est accessible en permanence
ETAPE II

Résultat de l'analyse un nuancier

\begin{tabular}{|c|c|c|}
\hline \multicolumn{3}{|c|}{$\begin{array}{c}\text { Prestation } \\
\text { Environnementale }\end{array}$} \\
\hline critère 1 & 0 & $\mathrm{~N}$ tous \\
\hline ritère 2 & 0 & $\mathrm{~N}$ tous \\
\hline ritère 3 & O & $\mathrm{N}$ tous \\
\hline critère 4 & O & $\mathrm{N}$ tous \\
\hline critère 5 & o & $\mathrm{N}$ tous \\
\hline critère 6 & o & $\mathrm{N}$ tous \\
\hline & & \\
\hline
\end{tabular}

ETAPE III Proposition de 3 nuanciers
ETAPE IV

Proposition de matériaux
ETAPE V

Sélection des informations
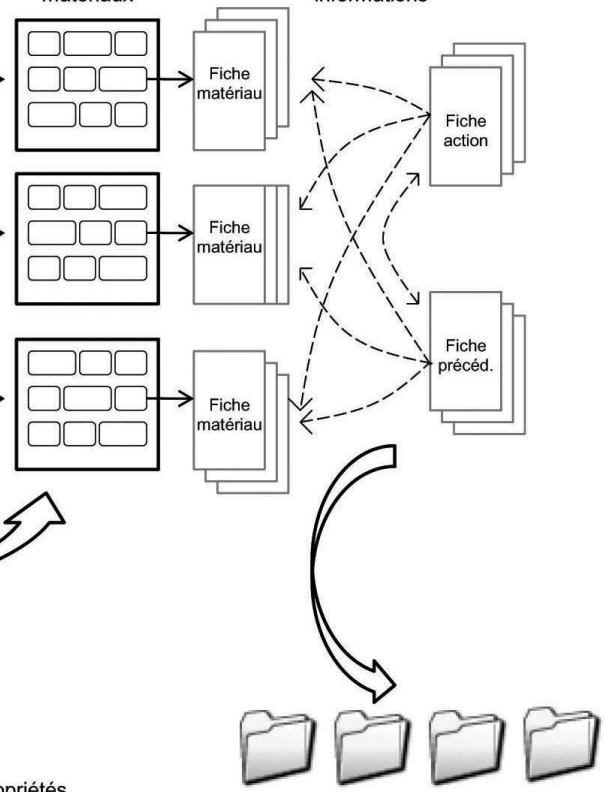

l'utilisateur crée ses propres fichiers en enregistrant les différentes fiches

Fig. 2.

Schéma de

la navigation

au sein de

Chroma. 
rique, présence de la végétation, masques existants et projetés, etc.). Puis le choix d'un élément, par exemple à l'échelle urbaine et paysagère, les traitements de sol, le mobilier urbain, murets, ou à l'échelle architecturale il peut s'agir d'une façade, d'une toiture, du traitement de sol mais ou encore à l'échelle de la pièce avec les revêtements muraux et de sol. En découle un court questionnaire qui décrit l'élément choisi et la sélection de matériaux. À partir de ces informations, Chrono étudie les effets des matériaux (brillance, teinte, chaux) froid, translucidité, etc.), leurs conséquences par temps ensoleillé, nuageux ou pluvieux, au fil des jours, des saisons voire des années (vieillissement des matériaux et salissures de façade). Les résultats sont visibles schématiquement sur les façades, plans, etc., et des diagrammes permettent de synthétiser les données. Chaque test fait l'objet d'un rapport qui réunit toutes les informations du projet, de son contexte environnant, la description de l'élément choisi, les fiches matériaux (qui notifient les dimensions écologiques, techniques et sensibles) et les schémas des effets des matériaux dans le temps. Ainsi il est possible de tester différentes volumétries, implantations, différents choix constructifs, différentes combinaison de matériaux, etc.
ETAPE I

Télécharger un fichier type .skt



ETAPE II

Saisies des données du projet
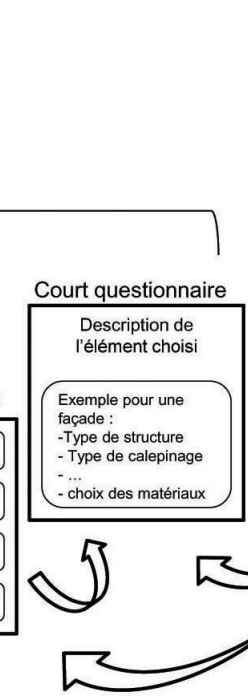

ETAPE III

Tests

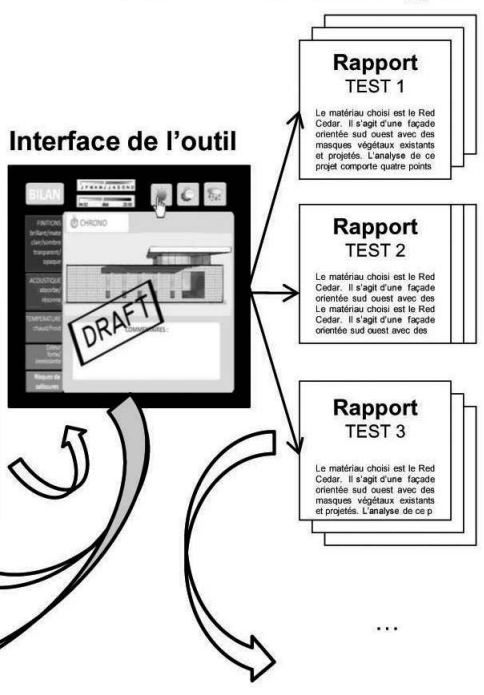



l'utilisateur crée ses propres fichiers en enregistrant les différentes fiches

Fig. 3.

Schéma de la navigation de Chrono. 
Chroma et Chrono sont, pour le moment au stade de concept. II s'agit d'une réflexion d'outil d'aide à la conception qui intègre les analyses de site et les choix des matériaux par une approche sensible. Nos travaux s'attachent à définir un cahier des charges d'outils idéaux en décrivant les données nécessaires (climatiques, techniques, etc.), leurs utilisations, imaginer la navigation au sein des informations, proposer des interfaces, commenter les résultats, leurs analyses, etc.

\section{Conclusion}

\section{Un outil destiné aux phases initiales de projet}

La composition de dilem'matériaux en trois outils de recherche initialement indépendants permet de proposer différentes pistes de réflexion pour instrumenter les choix de matériaux dès les premières phases de projet. La recherche par image est une proposition qui tente d'atténuer la carence d'information en phase de programmation, d'esquisse et d'avant-projet sommaire. Elle a pour objectif d'être une contribution pour la formulation d'un énoncé des intentions des équipes de concepteurs. Chroma et Chrono s'appuient sur l'analyse de site, ils développent davantage une logique de formulation de solutions. II s'agit de tester rapidement différentes variantes des projets.

Ces trois outils sont rassemblés par l'utilisation des fiches matériaux, actions et précédents. Leurs interactions, réalisées par des liens hypertextes, permettent une navigation où les équipes de concepteurs sont libres d'orienter leurs recherches selon leurs besoins, l'état d'avancement de leur réflexion. C'est un outil qui ambitionne d'accompagner les équipes de concepteurs au sein des questionnements, des quêtes d'information sur les matériaux de construction. II ne répond certainement pas à toutes les interrogations soulevées par le choix des matériaux. II est complémentaire aux outils et logiciels qui s'axent principalement sur les dimensions écologiques et techniques des matériaux.

\section{Les limites d'un tel outil}

Cet outil se heurte à un laborieux travail d'analyse de précédents. Pour être le plus performant possible il est essentiel de développer une riche base de données. La première phase consiste à sélectionner des précédents. Ils requièrent de dévoiler des qualités de matérialités hétéroclites, dans le même temps il est recommandé de rester vigilant sur l'analyse d'une gamme étendue de précédents, varier les usages, les partis architecturaux, urbains ou paysagers, les volumétries, les implantations, etc.

\section{Les développements envisagés}

Pourquoi ne pas imager de tels outils d'aide à la conception disponibles sur Internet. Ils seraient ainsi accessibles à tous, immédiatement et simultanément. Les logiciels libres stimulent la coopération, il est envisageable de créer un espace de discussion, d'échange sur le choix des matériaux. Actuellement, une maquette informatique de l'outil recherche par image est en cours, il s'agit de l'élaboration d'une base de données php sur serveur Internet. Parallèlement, cela pourrait aussi permettre aux équipes de concepteurs de proposer leurs projets comme précédents, ainsi la base de données de précédents pourrait s'auto-développer.

17. Google SketchUp est un logiciel de modélisation 3D, d'animation et de cartographie orienté vers l'architecture : http://sketchup.google.com/intl/fr/. 